\title{
Hounsfield units of the vertebral body and pedicle as predictors of pedicle screw loosening after degenerative lumbar spine surgery
}

\author{
${ }^{*} F e i$ Xu, MD, ${ }^{1,2}$ Da Zou, MD, ${ }^{1}$ Weishi Li, MD, ${ }^{1}$ Zhuoran Sun, MD, ${ }^{1}$ Shuai Jiang, MD, ${ }^{1}$ Siyu Zhou, MD, \\ and Zhuofu Li, MD1 \\ ${ }^{1}$ Orthopaedic Department, Peking University Third Hospital; and ${ }^{2}$ Peking University Health Science Center, Haidian District, \\ Beijing, China
}

\begin{abstract}
OBJECTIVE The authors aimed to compare the efficacy of lumbar vertebral body Hounsfield units (HUs) and pedicle $\mathrm{HUs}$ at predicting pedicle screw loosening.

METHODS The authors retrospectively assessed 143 patients with $L 3-5$ instrumentation. The patients were classified into one of two groups based on the status of their $\mathrm{L} 3$ screws (a screw loosening group or a control group). The pedicle HUs and vertebral HUs of $L 3$ were measured using preoperative lumbar CT scans, and the pedicle HUs were measured in two ways: by excluding or by including cortical bone.
\end{abstract}

RESULTS The screw loosening rate was 20.3\% $(n=29 / 143)$ at the 12-month follow-up. The vertebral body HUs and pedicle HUs in the screw loosening group were lower than those in the control group (vertebral body group: $98.6 \mathrm{HUs}$ vs $121.4 \mathrm{HUs}, p<0.001$; pedicle excluding cortical bone: $208.9 \mathrm{HUs}$ vs $290.5 \mathrm{HUs}, p=0.002$; pedicle including cortical bone: $249.4 \mathrm{HUs}$ vs $337.5 \mathrm{HUs}, p<0.001)$. The pedicle HUs tended to have a higher area under the receiver operating characteristic curve value in predicting screw loosening, compared with that of vertebral body HUs, but the difference was not statistically significant $(p>0.05)$. Among patients with low vertebral body HUs of $\leq 130$, the loosening rate was much lower in patients with pedicle HUs of $\leq 340$ than in those with pedicle $\mathrm{HUs}$ of $>340(31.0 \% \mathrm{vs} 13.0 \%$, respectively; $p<0.05)$.

CONCLUSIONS Vertebral body HUs alone are insufficient to accurately evaluate the risk of pedicle screw loosening. Therefore, it is important to collect both the pedicle $\mathrm{HU}$ and vertebral body $\mathrm{HU}$ measurements for surgical planning.

https://thejns.org/doi/abs/10.3171/2020.5.FOCUS20249

KEYWORDS CT Hounsfield units; pedicle bone density; screw loosening; vertebral bone density

$\mathrm{P}$ EDICLE screw loosening is one of the most common complications of lumbar posterior fixation. ${ }^{1-3}$ Some studies have found that the screw loosening rate was $1 \%-27 \%^{1,4,5}$ in normal patients and as high as $60 \%$ in osteoporotic patients. ${ }^{67}$ Röllinghoff et al. ${ }^{8}$ found that $20 \%$ of patients with screw loosening developed back pain, and screw loosening was one of the major reasons for revision surgery. ${ }^{9,10}$ In addition, many cases showed that screw loosening can result in other complications such as screw breakage, nonunion, pseudarthrosis, and kyphosis. ${ }^{11,12}$ Therefore, it is important to prevent loosening of the screws.
It is well known that osteoporosis is a risk factor for pedicle screw loosening. ${ }^{13-15}$ Therefore, preoperative bone mineral density (BMD) evaluation is critical for surgical planning. Previous studies found that the BMD of the vertebral body and the pedicle were closely related to the stability of fixation..$^{16-19}$ Compared with vertebral bone density, studies have shown that bone density of the pedicle has a greater impact on the stability of the pedicle screw, ${ }^{19}$ and the bone density of the intrapedicular segment is more important for the stability of the screw than the bone density of the screw tip segment and the segment close to the vertebral body. ${ }^{17,18}$ About $60 \%$ of pullout strength is de-

ABBREVIATIONS AUC = area under the curve; BMD = bone mineral density; DXA = dual-energy $x$-ray absorptiometry; $H U=$ Hounsfield unit; ROC = receiver operating characteristic; $\mathrm{ROI}=$ region of interest.

SUBMITTED March 28, 2020. ACCEPTED May 12, 2020.

INCLUDE WHEN CITING DOI: 10.3171/2020.5.FOCUS20249.

${ }^{*}$ F.X. and D.Z. contributed equally to this work. 

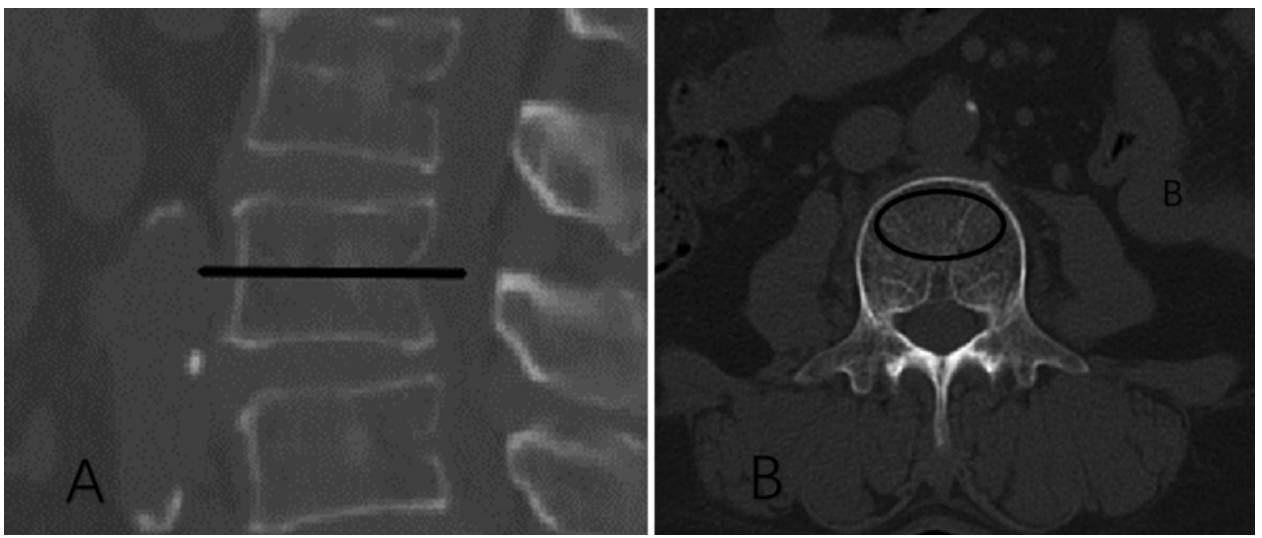

FIG. 1. The methods of measuring CT HU values of the vertebral body. At the level of the middle body, the vertebral body was equally divided into two parts (black line) in the craniocaudal direction at L3 (A). The vertebra's bone density was measured in the cancellous bone surrounded by an oval contour (black oval) excluding cortical bone and posterior venous plexus at the level of the middle body (B).

termined by the pedicle instead of the vertebral body. ${ }^{20}$ These may be due to the differences in the structure ${ }^{21}$ and the regional $\mathrm{BMD}^{17,18,22}$ between the pedicle and the vertebral body. Dual-energy x-ray absorptiometry (DXA) is the most common method for evaluating BMD and diagnosing osteoporosis. However, DXA might overestimate the BMD of the lumbar spine and miss osteoporosis in patients with lumbar degenerative disease, especially for degenerative lumbar scoliosis. ${ }^{23-25}$

Apart from DXA, the vertebral body Hounsfield unit (HU) value measured on clinical CT scans can also help detect osteoporosis. ${ }^{26-29}$ Several studies have shown that the HU value of the vertebral body is closely related to screw loosening. ${ }^{6,30-32}$ However, very few studies have investigated whether the $\mathrm{HU}$ value of the lumbar pedicle is related to screw loosening. ${ }^{19,31}$

This study was designed to compare the efficacy of using the HU values of two different regions-the vertebral body and the pedicle-in predicting screw loosening. Considering that screw loosening is the result of multiple factors ${ }^{33}$ we only assessed patients with L3 -5 instrumentation in this study and focused on the screw loosening found at L3 in order to reduce the influence of potential factors other than CT HUs.

\section{Methods \\ Patients}

This study was approved by the institutional review board of our hospital. The need to obtain informed consent was waived due to the retrospective nature of the study. A total of 143 patients who underwent primary transpedicular lumbar fixation for lumbar degenerative diseases from July 2011 to December 2017 were analyzed retrospectively. The inclusion criteria were as follows: 1) instrumentation from L3 to L5 in patients aged $>50$ years at the time of surgery; 2) follow-up data for at least 12 months; and 3) and lumbar CT scans obtained within 3 months before the surgery. To exclude other confounders as much as possible, we established the following exclusion criteria: 1) history of congenital spinal deformity, spinal trauma, spinal tu- mor, spinal tuberculosis, ankylosing spondylitis, or previous spinal surgery; 2) presence of metabolic bone disease or long-term use of medicines like corticosteroids, which can influence BMD; and 3) patients who underwent revision surgery due to non-screw loosening causes, such as intraoperative nerve injury, postoperative wound hematoma, and adjacent disc degeneration in the 12 months after primary surgery. Screw loosening was defined as a clear zone of minimal thickness of $\geq 1 \mathrm{~mm}$ around the pedicle screw on radiography. ${ }^{9,30,32}$ A senior spine surgeon (Z.S.) independently evaluated the radiographs. All patients' surgeries were planned and performed by the same surgical team. At the 12-month follow-up visit, patients exhibiting screw loosening at L3 were assigned to the screw loosening group. The remaining patients were assigned to the control group.

\section{Estimation of Bone Density}

All patients underwent preoperative lumbar CT (Definition, Siemens). The tube voltage of the $\mathrm{CT}$ scans was set at $120 \mathrm{kV}$. The average of the region of interest (ROI) HU value was calculated using a PACS. An axial plane at the mid-vertebral body was chosen for the measurement of vertebral body HU (Fig. 1). The ROI was chosen to include as much trabecular bone as possible and to avoid cortical bone and heterogeneous areas, such as the posterior venous plexus, bone island, and compressed bone. The diameter of the pedicle was also measured at the narrowest part of the L3 pedicle (Fig. 2). Additionally, the $\mathrm{HU}$ of the pedicle was similarly measured on the axial plane of the mid-pedicle of L3. ROIs were chosen either by exclusion of the cortical bone or inclusion of the cortical bone on both sides. In addition, the pedicle ROI was placed at the narrowest part of the L3 pedicle, as shown in Fig. 2. The pedicle HU was the average of the HU values of both sides.

\section{Statistical Analysis}

Statistical analysis was performed using SPSS version 23 (IBM Corp.) and MedCalc Statistical Software version 

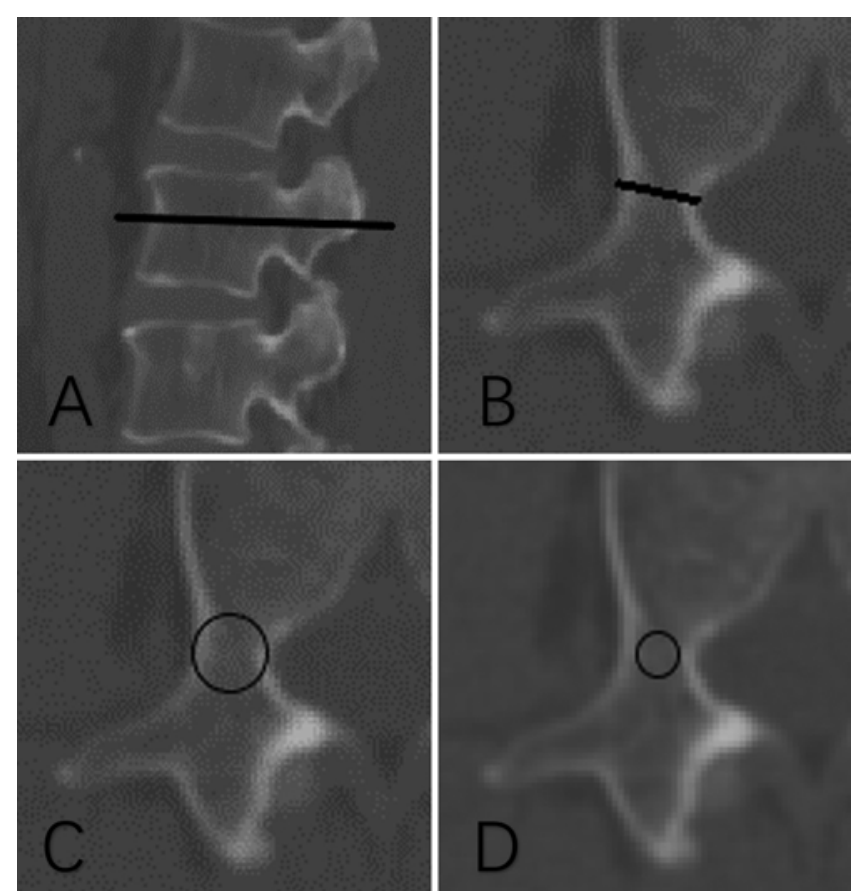

FIG. 2. The methods of measuring the diameter and CT HU values of the pedicle. At the middle level of the pedicle (black line) in a parasagittal section on a CT scan at L3 (A), the axial CT plane was chosen to measure the diameter of the pedicle and pedicle $\mathrm{HU}$. The diameter of pedicle was measured at the narrowest part of the $\mathrm{L} 3$ pedicle (black line) including the cortex (B). The pedicle ROls were placed as circles at the narrowest part of the $\mathrm{L} 3$ pedicle on the bilateral sides including the cortical bone (C) and excluding the cortical bone (D).

15.6.1 (MedCalc Software). The independent-samples Student t-test was used for continuous variables. Chi-square testing was used for categorical data. Using MedCalc Statistical Software, the vertebral body HU, the HU values of the pedicle excluding cortical bone and the pedicle including cortical bone, and the diameter of pedicle were analyzed in association with screw loosening using receiver operating characteristic (ROC) curves. Logistic regression analysis was performed to determine which parameter was independently associated with screw loosening.

\section{Results \\ Demographics}

The screw loosening rate was $20.3 \%(29 / 143)$ at L3 in the patients with L3-5 instrumentation. Compared with the control group, patients' BMI values in the screw loosening group were significantly higher $(\mathrm{p}=0.049)$, but no significant differences in sex or age between the two groups was found (Table 1).

\section{HU Values of Vertebral Body and Pedicle}

A comparison of $\mathrm{HU}$ values of the vertebral body and pedicle between the two groups is listed in Table 1. The Pearson correlation coefficient between CT HU values of the vertebral body and the pedicle excluding and including the cortex was $0.409(\mathrm{p}<0.001)$ and $0.456(\mathrm{p}<$ $0.001)$, respectively. The CT HU values of the L3 verte-
TABLE 1. Demographic characteristics and BMD

\begin{tabular}{lccc}
\hline \multicolumn{1}{c}{ Characteristic } & $\begin{array}{c}\text { Screw Loosening } \\
\text { Group }\end{array}$ & $\begin{array}{c}\text { Control } \\
\text { Group }\end{array}$ & $\begin{array}{c}\mathrm{p} \\
\text { Value }\end{array}$ \\
\hline Age (yrs) & $62.0 \pm 6.7$ & $62.0 \pm 6.4$ & 0.934 \\
\hline Male/female ratio & $16: 13$ & $45: 69$ & 0.127 \\
\hline BMl (kg/m ${ }^{2}$ ) & $26.9 \pm 2.9$ & $25.7 \pm 3.3$ & 0.049 \\
\hline Mean pedicle diameter & $10.3 \pm 1.9$ & $9.3 \pm 1.9$ & 0.008 \\
\hline $\begin{array}{l}\text { L3 vertebral body CT } \\
\text { HU value }\end{array}$ & $98.6 \pm 25.8$ & $121.4 \pm 39.7$ & $<0.001$ \\
\hline $\begin{array}{l}\text { L3 pedicle CT HU value } \\
\text { (excluding cortical bone) }\end{array}$ & $208.9 \pm 69.5$ & $290.5 \pm 132$ & 0.002 \\
\hline $\begin{array}{l}\text { L3 pedicle CT HU value } \\
\text { (including cortical bone) }\end{array}$ & $249.4 \pm 71.4$ & $337.5 \pm 125.5$ & 0.001 \\
\hline
\end{tabular}

bral body and pedicle (excluding and including cortical bone) in the screw loosening group were lower than those in the control group (vertebral HU value: $\mathrm{p}<0.001$; pedicle HU value excluding the cortical bone: $\mathrm{p}=0.002$; and pedicle HU value including the cortical bone: $p<0.001$ ). The mean pedicle diameters in the screw loosening group were higher than those in the control group $(p=0.008)$. According to the paired t-test, the pedicle HU value including the cortical bone was significantly higher than that of the pedicle HU value excluding the cortical bone (319.6 \pm 121.7 vs $274.0 \pm 126.1, \mathrm{p}<0.001)$.

\section{ROC Curve Analysis}

We established the ROC curve to evaluate the validity of using CT HUs to predict screw loosening. They are shown in Table 2. The pedicle CT HUs (excluding and including cortical bone) had larger areas under the curve (AUCs) than those of the vertebral CT HUs, but there was no significant difference $(\mathrm{p}>0.05)$.

\section{BMD Analysis of Screw Loosening}

According to the ROC analysis, we identified the HU cutoff (take a multiple of 10) for identifying high-risk patients for screw loosening. The cutoff value of high sensitivity (about 90\%) was set at $130 \mathrm{HUs}$ for the vertebral body and $340 \mathrm{HUs}$ for the pedicle (including cortical bone). The cutoff value for high specificity (about 90\%) was set at 70 HUs for the vertebral body and 180 HUs for the pedicle. We divided the patients into 4 groups accord-

TABLE 2. ROC analysis of CT HU values of the vertebral body and pedicle in predicting screw loosening

\begin{tabular}{lccc}
\hline \multicolumn{1}{c}{ Characteristic } & AUC & AUC: $95 \% \mathrm{Cl}$ & $\mathrm{p} \mathrm{Value}$ \\
\hline $\begin{array}{l}\text { Mean L3 pedicle CT HU value } \\
\text { (excluding cortical bone) }\end{array}$ & 0.689 & $0.607-0.764$ & 0.002 \\
\hline $\begin{array}{l}\text { Mean L3 pedicle CT HU value } \\
\text { (including cortical bone) }\end{array}$ & 0.721 & $0.639-0.792$ & $<0.001$ \\
\hline Mean diameter of pedicles & 0.654 & $0.570-0.731$ & 0.011 \\
\hline $\begin{array}{l}\text { L3 vertebral body CT HU value } \\
\text { (middle body) }\end{array}$ & 0.674 & $0.590-0.750$ & 0.004 \\
\hline
\end{tabular}


TABLE 3. Thresholds of CT HU values of the pedicles and vertebral bodies

\begin{tabular}{ccc}
\hline Screw Loosening Rate* & $\begin{array}{c}\text { CT HU of VBs } \\
\leq 130\end{array}$ & $\begin{array}{c}\text { CT HU of VBs } \\
>130\end{array}$ \\
\hline CT HUs of the pedicle $\leq 340$ & $31.0 \%(22 / 71)$ & $17.6 \%(3 / 17)$ \\
\hline CT HUs of the pedicle $>340$ & $13.0 \%(3 / 23)$ & $3.1 \%(1 / 32)$ \\
\hline
\end{tabular}

$\mathrm{VB}=$ vertebral body.

${ }^{*}$ Number of screw loosening/total number.

ing to the cutoff value of high sensitivity and listed the corresponding screw loosening rates (Table 3 ).

\section{Logistic Regression of Risk Factors and Screw Loosening}

We entered the risk factors with a $\mathrm{p}$ value $<0.1$ into logistic regression, including BMI, mean diameter of the pedicle, and CT HU. Since vertebral body HUs had significant linear correlation with pedicle HUs, they were not put into the regression model at the same time. As for the regression analysis including BMI, pedicle diameter, and a vertebral body $\mathrm{HU}$ of $\leq 130$, the following results were found: BMI, OR 1.192, 95\% CI 1.020-1.393, p = 0.027; pedicle diameter, OR 1.349, 95\% CI 1.062-1.713, $\mathrm{p}=$ 0.014; and vertebral body $\mathrm{HU}$ of $\leq 130$, OR $6.555,95 \% \mathrm{CI}$ $1.770-24.272, p=0.005$. As for the regression analysis including BMI, pedicle diameter, and pedicle $\mathrm{HU}$ of $\leq 340$, the following results were found: BMI, OR 1.171, 95\% CI 1.013-1.353, $\mathrm{p}=0.032$; pedicle diameter, OR $1.287,95 \%$ CI 1.014-1.633, $\mathrm{p}=0.038$; and pedicle HU of $\leq 340$, OR $4.848,95 \%$ CI $1.520-15.465, \mathrm{p}=0.008$.

\section{Discussion}

This study found that both the CT HU values of the pedicle and the vertebral body were closely related to screw loosening. The lower the CT HU values, the higher the possibility of screw loosening. Furthermore, patients with a low vertebral body $\mathrm{HU}$ value are not always at a high risk of screw loosening, especially when they have a high lumbar pedicle HU value.

It is well known that pedicle screw loosening can arise from many factors other than BMD, such as the vertebral level of screws, length of fixation, and so on. To decrease the influence of confounding factors, this study only chose patients with L3-5 instrumentation, focusing on the status of pedicle screws at L3.

In this study, the screw loosening rate was up to $20.3 \%$ ( $n=29 / 143$ ), which is comparable with the results of previous studies. ${ }^{1,4,5}$ We found that patients with a high BMI were at a higher risk of screw loosening. Furthermore, we found that the mean diameter of pedicles in the control group was smaller than that of pedicles in the screw loosening group. According to the logistic regression, the pedicle diameter was also an independent predictor of screw loosening. It may be related to the fact that the screw gets more pullout strength when it is close to the cortical bone. However, in osteoporotic patients, some studies have found that a larger-diameter screw does not increase its stability. ${ }^{34}$
Recently, the CT HU value has been widely used for the evaluation of BMD and screening for osteoporosis. ${ }^{26-29}$ A few studies have proven that the CT HU value of the vertebral body is closely related to screw loosening. . $^{6,30-32}$ It is well known that most of the pullout strength is determined by the lumbar pedicle instead of the vertebral body. However, there are a few studies investigating the effect of the pedicle CT HU on screw loosening. ${ }^{19,31}$

In this study, we found that the CT HU values of both the pedicle and the vertebral body were significantly lower in the screw loosening group than that in the control group. Owing to different trabecular architecture and a higher biomechanical relevance, it was reported that the intrapedicular segment is more important for the stability of screw than the segment of the screw tip and the segment closing to the vertebral body. ${ }^{17,18}$ In agreement with previous studies, we found that the pedicle $\mathrm{HU}$ tended to have a higher AUC in predicting screw loosening than the vertebral body HU. However, there was no statistically significant difference. The possible reason is that the vertebral body $\mathrm{HU}$ is positively correlated to the pedicle $\mathrm{HU}$ (excluding cortex: $\mathrm{R}=0.409, \mathrm{p}<0.001$; including cortex: $\mathrm{R}=0.456, \mathrm{p}<0.001)$. The screw loosening rate of patients with the vertebral body $\mathrm{HU}$ of $\leq 130$ and pedicle HU (including cortical bone) of $\leq 340 \mathrm{HU}$ is much higher than patients with the vertebral CT HU of $\leq 130$ and pedicle HU (including cortical bone) of $>340$ (31.0\% vs $13.0 \%$, $\mathrm{p}<0.05)$. This proves that low vertebral body HU is not always associated with high risk of screw loosening, especially for patients with high pedicle HU. Hence, it is important to consider the BMD of both the pedicle and the vertebral body when making the preoperative evaluation.

A previous study has collected the BMD of the screw's pathway to predict screw loosening by 3D image analysis software. ${ }^{31}$ However, on axial images of the vertebral body, the screw pathway's CT HU value cannot be measured directly with routine PACS. If we focus on the CT $\mathrm{HU}$ value of the screw's pathway, the measurement may be complicated and cannot be applied in routine clinical practice. Thus, we opted to use the routine method of measuring the preoperative lumbar CT HU values of the vertebral body and the pedicle to predict screw loosening, which is convenient and simple.

There are two main limitations to this study. As this was a retrospective study, further prospective studies are warranted to validate our findings. Next, this study only focused on the HU of L3. However, the CT HU values of the vertebral body and the pedicle may be different at different vertebral levels, leading to different threshold values for predicting screw loosening. Further research is recommended in this regard.

\section{Conclusions}

Both pedicle and vertebral body CT HU values are associated with screw loosening, which can be regarded as indicators of screw stability. Moreover, the lower the CT HU values are, the higher the risk of screw loosening. The pedicle CT HU tended to have a better ability in predicting screw loosening than the vertebral body CT HU. In addition to vertebral body BMD, assessment of the pedicle 
BMD using CT may potentially improve preoperative surgical planning.

\section{Acknowledgments}

This study was supported by the Clinical Cohort Construction Program of Peking University Third Hospital (BYSYDL2019006) and the National Natural Science Foundation of China (8187090666).

\section{References}

1. Galbusera F, Volkheimer D, Reitmaier S, et al. Pedicle screw loosening: a clinically relevant complication? Eur Spine J. 2015;24(5):1005-1016.

2. Halvorson TL, Kelley LA, Thomas KA, et al. Effects of bone mineral density on pedicle screw fixation. Spine (Phila Pa 1976). 1994;19(21):2415-2420.

3. Soshi S, Shiba R, Kondo H, Murota K. An experimental study on transpedicular screw fixation in relation to osteoporosis of the lumbar spine. Spine (Phila Pa 1976). 1991;16(11): $1335-1341$

4. Xie Y, Ma H, Li H, et al. Comparative study of unilateral and bilateral pedicle screw fixation in posterior lumbar interbody fusion. Orthopedics. 2012;35(10):e1517-e1523.

5. Uehara M, Takahashi J, Ikegami S, et al. Pedicle screw loosening after posterior spinal fusion for adolescent idiopathic scoliosis in upper and lower instrumented vertebrae having major perforation. Spine (Phila Pa 1976). 2017;42(24):18951900.

6. Bredow J, Boese CK, Werner CM, et al. Predictive validity of preoperative CT scans and the risk of pedicle screw loosening in spinal surgery. Arch Orthop Trauma Surg. 2016; 136(8):1063-1067.

7. El Saman A, Meier S, Sander A, et al. Reduced loosening rate and loss of correction following posterior stabilization with or without PMMA augmentation of pedicle screws in vertebral fractures in the elderly. Eur J Trauma Emerg Surg. 2013;39(5):455-460.

8. Röllinghoff M, Schlüter-Brust K, Groos D, et al. Mid-range outcomes in 64 consecutive cases of multilevel fusion for degenerative diseases of the lumbar spine. Orthop Rev (Pavia). 2010;2(1):e3.

9. Kocak T, Cakir B, Reichel H, Mattes T. Screw loosening after posterior dynamic stabilization-review of the literature. Acta Chir Orthop Traumatol Cech. 2010;77(2):134-139.

10. Wu JC, Huang WC, Tsai HW, et al. Pedicle screw loosening in dynamic stabilization: incidence, risk, and outcome in 126 patients. Neurosurg Focus. 2011;31(4):E9.

11. Berjano P, Bassani R, Casero G, et al. Failures and revisions in surgery for sagittal imbalance: analysis of factors influencing failure. Eur Spine J. 2013;22(suppl 6):S853-S858.

12. McLain RF, Sparling E, Benson DR. Early failure of shortsegment pedicle instrumentation for thoracolumbar fractures. A preliminary report. J Bone Joint Surg Am. 1993;75(2): 162-167.

13. Varghese V, Saravana Kumar G, Krishnan V. Effect of various factors on pull out strength of pedicle screw in normal and osteoporotic cancellous bone models. Med Eng Phys. 2017;40:28-38.

14. Okuyama K, Abe E, Suzuki T, et al. Influence of bone mineral density on pedicle screw fixation: a study of pedicle screw fixation augmenting posterior lumbar interbody fusion in elderly patients. Spine J. 2001;1(6):402-407.

15. Amirouche F, Solitro GF, Magnan BP. Stability and spine pedicle screws fixation strength - A comparative study of bone density and insertion angle. Spine Deform. 2016;4(4): 261-267.

16. Inceoğlu S, Kilinçer C, Tami A, McLain RF. Cortex of the pedicle of the vertebral arch. Part I: Deformation characteristics during screw insertion. J Neurosurg Spine. 2007;7(3): 341-346.

17. Hirano T, Hasegawa K, Takahashi HE, et al. Structural characteristics of the pedicle and its role in screw stability. Spine (Phila Pa 1976). 1997;22(21):2504-2510.

18. Zhang QH, Tan SH, Chou SM. Investigation of fixation screw pull-out strength on human spine. J Biomech. 2004;37(4): 479-485.

19. Wichmann JL, Booz C, Wesarg S, et al. Quantitative dualenergy CT for phantomless evaluation of cancellous bone mineral density of the vertebral pedicle: correlation with pedicle screw pull-out strength. Eur Radiol. 2015;25(6): $1714-1720$

20. Weinstein JN, Rydevik BL, Rauschning W. Anatomic and technical considerations of pedicle screw fixation. Clin Orthop Relat Res. 1992;(284):34-46.

21. Lehman RA Jr, Helgeson MD, Dmitriev AE, et al. What is the best way to optimize thoracic kyphosis correction? A micro-CT and biomechanical analysis of pedicle morphology and screw failure. Spine (Phila Pa 1976). 2012;37(19):E1171E1176.

22. Defino HL, Vendrame JR. Role of cortical and cancellous bone of the vertebral pedicle in implant fixation. Eur Spine J. 2001;10(4):325-333.

23. Choi MK, Kim SM, Lim JK. Diagnostic efficacy of Hounsfield units in spine CT for the assessment of real bone mineral density of degenerative spine: correlation study between T-scores determined by DEXA scan and Hounsfield units from CT. Acta Neurochir (Wien). 2016;158(7):1421-1427.

24. Pappou IP, Girardi FP, Sandhu HS, et al. Discordantly high spinal bone mineral density values in patients with adult lumbar scoliosis. Spine (Phila Pa 1976). 2006;31(14):16141620.

25. Muraki S, Yamamoto S, Ishibashi H, et al. Impact of degenerative spinal diseases on bone mineral density of the lumbar spine in elderly women. Osteoporos Int. 2004;15(9):724-728.

26. Zou D, Li W, Deng C, et al. The use of CT Hounsfield unit values to identify the undiagnosed spinal osteoporosis in patients with lumbar degenerative diseases. Eur Spine J. 2019; 28(8) 1758-1766

27. Hendrickson NR, Pickhardt PJ, Del Rio AM, et al. Bone mineral density T-scores derived from CT attenuation numbers (Hounsfield units): clinical utility and correlation with dual-energy x-ray absorptiometry. Iowa Orthop J. 2018;38: 25-31.

28. Anderson PA, Polly DW, Binkley NC, Pickhardt PJ. Clinical use of opportunistic computed tomography screening for osteoporosis. J Bone Joint Surg Am. 2018;100(23):2073-2081.

29. Lee SJ, Binkley N, Lubner MG, et al. Opportunistic screening for osteoporosis using the sagittal reconstruction from routine abdominal CT for combined assessment of vertebral fractures and density. Osteoporos Int. 2016;27(3):1131-1136.

30. Zou D, Muheremu A, Sun Z, et al. Computed tomography Hounsfield unit-based prediction of pedicle screw loosening after surgery for degenerative lumbar spine disease. J Neurosurg Spine. 2020;32(5):716-721.

31. Sakai Y, Takenaka S, Matsuo Y, et al. Hounsfield unit of screw trajectory as a predictor of pedicle screw loosening after single level lumbar interbody fusion. J Orthop Sci. 2018; 23(5):734-738.

32. Zou D, Sun Z, Zhou S, et al. Hounsfield units value is a better predictor of pedicle screw loosening than the T-score of DXA in patients with lumbar degenerative diseases. Eur Spine J. 2020;29(5):1105-1111.

33. Kim JB, Park SW, Lee YS, et al. The effects of spinopelvic parameters and paraspinal muscle degeneration on S1 screw loosening. J Korean Neurosurg Soc. 2015;58(4):357-362.

34. Brantley AG, Mayfield JK, Koeneman JB, Clark KR. The 
effects of pedicle screw fit. An in vitro study. Spine (Phila $\mathrm{Pa}$ 1976). 1994;19(15):1752-1758.

\section{Disclosures}

The authors report no conflict of interest concerning the materials or methods used in this study or the findings specified in this paper.

\section{Author Contributions}

Conception and design: W Li, Xu, Zou. Acquisition of data: Xu, Zou, Sun, Jiang, Zhou. Analysis and interpretation of data: W Li,
Xu, Zou, Sun, Z Li. Drafting the article: Xu, Sun, Jiang. Critically revising the article: Xu, Zou, Sun, Zhou. Reviewed submitted version of manuscript: $\mathrm{W} \mathrm{Li}, \mathrm{Xu}$, Jiang. Approved the final version of the manuscript on behalf of all authors: W Li. Statistical analysis: $\mathrm{Xu}$, Zou, Jiang. Administrative/technical/material support: W Li, Xu. Study supervision: W Li, Xu.

\section{Correspondence}

Weishi Li: Peking University Third Hospital, Beijing, China. puh3liweishi@163.com. 